MITSUBISHI ELECTRIC RESEARCH LABORATORIES

http://www.merl.com

\title{
Inter-Cell Interference Management in WiMAX Downlinks by A Stackelberg Game Between BSs
}

\author{
Yingda Chen, Koon Hoo Teo, Shalinee Kishore, Jinyun Zhang
}

TR2008-059 September 2008

\begin{abstract}
We develop a game theoretic framework for effective and adaptive inter-cell interference management in the OFDMA-based WiMAX/3GPP-LTE systems. Such approach requires no fixed spectrum planning beforehand and does not affect the spectrum usage for users that are not subject to inter-cell interference. Based on the handoff mechanisms in WiMAX systems, we also examine means of categorizing users who are, or are not, subject to potential interference. Different optimization opportunities for BSs are identified and analyzed. The interactions between BSs are modeled by a Stackelberg game in which BSs can make intelligent and rational decisions to reduce interference with minimum optimization cost. The algorithm to attain stable Nash Equilibrium is investigated and the behaviors of BSs with different preferences in the game are examined
\end{abstract}

ICC 2008

This work may not be copied or reproduced in whole or in part for any commercial purpose. Permission to copy in whole or in part without payment of fee is granted for nonprofit educational and research purposes provided that all such whole or partial copies include the following: a notice that such copying is by permission of Mitsubishi Electric Research Laboratories, Inc.; an acknowledgment of the authors and individual contributions to the work; and all applicable portions of the copyright notice. Copying, reproduction, or republishing for any other purpose shall require a license with payment of fee to Mitsubishi Electric Research Laboratories, Inc. All rights reserved.

Copyright (C) Mitsubishi Electric Research Laboratories, Inc., 2008

201 Broadway, Cambridge, Massachusetts 02139 



\title{
Inter-Cell Interference Management in WiMAX Downlinks by A Stackelberg Game between BSs
}

\author{
Yingda Chen ${ }^{\dagger}$, Koon Hoo $\mathrm{Teo}^{\ddagger}$, Shalinee Kishore ${ }^{\dagger}$ and Jinyun Zhang ${ }^{\ddagger}$ \\ ${ }^{\dagger}$ Electrical and Computer Engineering, Lehigh University, Bethlehem, PA 18015 \\ ${ }^{\ddagger}$ Mitsubishi Electric Research Laboratories, Cambridge, MA 02139 \\ $\dagger\{$ Yingda,Skishore $\} @$ Lehigh.edu, ${ }^{\ddagger}\{$ Teo, Jzhang $\} @$ MERL.com
}

\begin{abstract}
We develop a game theoretic framework for effective and adaptive inter-cell interference management in the OFDMA-based WiMAX/3GPP-LTE systems. Such approach requires no fixed spectrum planning beforehand and does not affect the spectrum usage for users that are not subject to inter-cell interference. Based on the handoff mechanisms in WiMAX systems, we also examine means of categorizing users who are, or are not, subject to potential interference. Different optimization opportunities for BSs are identified and analyzed. The interactions between BSs are modeled by a Stackelberg game in which BSs can make intelligent and rational decisions to reduce interference with minimum optimization cost. The algorithm to attain stable Nash Equilibrium is investigated and the behaviors of BSs with different preferences in the game are examined.
\end{abstract}

\section{INTRODUCTION}

The ever-growing needs for broadband wireless access have recently provoked the development of the new IEEE 802.16 standards [1][2]. This emerging family of IEEE standards, usually known by its industry consortium, WiMAX(Worldwide Interoperability for Microwave Access), promises to support high-rate wireless data services comparable to wire-line connections. WiMAX systems adopt Orthogonal Frequency Division Multiple Access (OFDMA) for serving multiple mobile stations (MSs) [2]. In such systems, the intra-cell interference can be minimized as long as orthogonality among subcarriers is properly maintained, which is quite feasible with appropriate scheduling in a connection-oriented system like WiMAX. Suppression of inter-cell (co-channel) interference, however, is more challenging, and is especially important to the MSs located at the cell boundary, when full spectrum reuse is implemented.

In recognition of its importance, different schemes have been proposed to mitigate inter-cell interference in OFDMAbased systems [4]-[7]. However, most of such schemes rely strongly on careful system-wise frequency planning. For example, in soft frequency reuse [3], the spectrum is divided into multiple chunks and then allocated to MSs located at the center and edge of the cell. The base stations (BSs) of adjacent cells coordinate beforehand and agree on the allocations of these frequency chunks, to ensure that the "edge spectrum chunks" of adjacent cells differ. In partial frequency reuse [4][5], frequency reuse factor is set to be 1 for cell center MSs and 3 for cell edge MSs, which inevitably necessitates strict frequency planning. Performances of those two different schemes are compared in [6]. The spectrum-usage planning aforementioned usually requires a super centralized control above different BSs, which is even more difficult to implement when the BSs belong to different wireless operators. In addition, planning before system deployment can suffer from severe inefficiency when imbalances exist in the distribution of the MSs (e.g., when most MSs are located at the cell edge). Moreover, full spectrum reuse cannot be maintained in some of these schemes (e.g., the partial frequency reuse). Various other inter-cell interference management schemes for OFDMA systems are being proposed as well, yet most of them stem from the channel assignment schemes for conventional cellular systems [8] and usually require system-wise planning as well.

In this paper, we propose an adaptive protocol to suppress inter-cell interference (a.k.a. subcarrier collisions). While certain aspects of the protocol are designed specifically for the WiMAX downlinks, the underlying methodology can be applied to general broadband systems. The proposed protocol requires no preset spectrum planning and does not require users that are not subject to interference to coordinate their spectrum usage with those who are. Different optimization opportunities for interference suppression are identified and analyzed. The interactions between BSs are modeled as a Stackelberg leader-follower game in which rational BSs seek to reduce interference with minimum cost. We also develop the algorithm for implementing such game and analyze the behaviors of BSs with different preferences. Immediately following this introduction is a system description in Section II. The Stackelberg game between BSs is presented in Section III, which also includes the methodology for modeling BSs' utility functions and the analysis of different game outcomes. Finally, we conclude our discussion in Section IV.

\section{SYSTEM DESCRIPTION}

We consider in this paper the downlink interference management for a two-cell system (extension to multi-cell system is straightforward, e.g., a multi-cell system can be modeled by allowing a BS to involve in multiple two-player games with different adjacent BSs) shown in Fig. 1. Each circular cell has radius $R$ which overlap $\alpha R(\alpha \in[0,1])$ with each other.

The MSs in each cell are assumed to be uniformly distributed. We also assume that only MSs in overlapping region are subject to interference and refer to such region as the interference zone (IZ), the MSs from cell $i$ located in IZ form the set $\mathcal{I}_{i}{ }^{1}$. Accordingly, the MSs outside IZ form the set $\mathcal{I}_{i}^{C}$.

\footnotetext{
${ }^{1}$ Such retain assumption and simplification is taken here only for analytical and notational convenience. When the MSs are not uniformly distributed, or when some MSs outside the overlapping area are subject to co-channel interference as well, the methodologies proposed herein still apply. The only difference lies in the definition of IZ and $\mathcal{I}_{i}$, which may lack an readily intuitive interpretation but does not affect the proposed protocol otherwise.
} 
In addition, assuming that the number of subcarriers allocated to a MS is uniformly distributed as well, when the number of MSs in a cell is substantially-large, the geographic distribution of subcarrier usage is also uniformly distributed within a cell.We assume full spectrum reuse and that the spectrum in each cell is divided into $S$ subcarriers which form the set $\mathcal{S}$. The fraction of the overlapping area can be computed as:

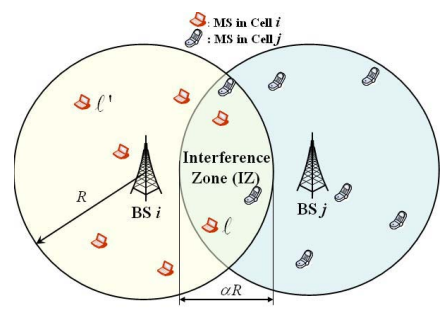

$$
f(\alpha)=\frac{2}{\pi} \cdot\left[\begin{array}{c}
\text { Fig. 1: Two Cell Model } \\
\arccos \left(1-\frac{\alpha}{2}\right)-\left(1-\frac{\alpha}{2}\right)
\end{array} \sqrt{\left(4 \alpha-\alpha^{2}\right)}\right] .
$$

\section{A. Identifying the MSs in IZ}

Unless in the unlikely case that each MS is equipped with GPS, the BS may not be able to obtain the exact realtime location of a MS. In this paper, we design a protocol to facilitate the identification of the set $\mathcal{I}_{i}$ based on the handoff overhead. Specifically, for mobile WiMAX system, the IEEE 802.16e [2] specifies handoff mechanisms using fast base station switching (FBSS) or macro diversity hand over (MDHO). We focus the discussion in this paper on FBSS, but the methodologies described here apply to MDHO and other handoff mechanisms in systems like 3GPP-LTE as well.

With FBSS, a MS $\ell$ maintains an active set (also known as the diversity set) which includes the BSs that MS can (possibly reliably) communicate with. Within the set, the BS to which MS is currently registered is marked as "anchor" BS. While FBSS was originally designed to facilitate seamless handoff, the BSs in the active set (other than the anchor BS) are at the same time the BSs that can cause possible downlink interference to the MS $\ell$. In the scheme shown in Fig. 1, when $\ell$ (registered with BS $i$ ) is located in IZ, its active set would be $\{i(A), j\}$, which indicates that BS $i$ is the anchor(A) BS of $\ell$ and $j$ is a BS that 1) $\ell$ can communicate with, from the perspective of handoff and 2 ) could cause interference to $\ell$, from the perspective of interference management ${ }^{2}$. In FBSS protocol, the content of active set is feedback to all BSs in the set. In this way, when BS $i$ learns that the active set of $\ell$ is $\{i(A), j\}$, it can determine that $\ell \in \mathcal{I}_{i \cap j}$, which indicates that $\ell$ is in the IZ and $\ell$ is subject to interference from downlink transmissions in cell $j$. For the two-cell model considered here, we will use the simplified notation $\ell \in \mathcal{I}_{i}$ to indicate that $\ell$ is subject to inter-cell interference. In contrast, when a MS $\ell^{\prime}$ registered with BS $i$ has an active set $\{i(A)\}$, BS $i$ can determine that $\ell^{\prime}$ is located outside its IZ.

${ }^{2}$ In FBSS, MS monitors CINRs from different BSs and make intelligence decisions as to whether a BS should be included in the active set. Detailed specifications of the maintenance of an active set at MS are given in [2]. In our application, the threshold CINR can be modified to determine whether a BS will cause interference to a MS. Generally, the threshold CINR $\left(\gamma_{T}^{i}\right)$ used for identifying potential interfering BSs should be lower than the CINR $\left(\gamma_{T}^{h}\right)$ used for identifying BSs for handover. For example, to guarantee the detection of all potential interfering BSs, we expect $\gamma_{T}^{i} \leq \gamma_{T}^{h}-K \mathrm{~dB}$. In practice, value of $K$ usually ranges from 6 to 10 and should be determined upon specific system configurations.

\section{B. Partial Information Exchange}

Since the MSs outside IZ are not subject to inter-cell interference, we allow the subcarrier allocation to these MSs to proceed randomly without any coordination between cells. After the subcarriers have been allocated to MSs in $\mathcal{I}_{i}^{C}$, the remaining subcarriers in cell $i$ are denoted by the set $\mathcal{S}_{i}^{A} \subset \mathcal{S}$. BSs $i$ and $j$ then exchange (through the backbone network or direct wireless links) information on $\mathcal{S}_{i}^{A}$ and $\mathcal{S}_{j}^{A}$ to facilitate interference management. Note that only partial information on the subcarrier usage (i.e., $\mathcal{S}_{i}^{A}$ and $\mathcal{S}_{j}^{A}$ ) need to be exchanged between BSs, the subcarrier allocation to MSs outside IZ is not affected. In this way, we do not have to rely on any planning of subcarrier allocation beforehand, and do not restrict subcarrier usage to a certain fraction of MSs (which is the case in partial and soft spectrum reuse studied in [3]-[6]). Also note that with the absence of "super-BS" that coordinates operations of different BSs (e.g., when different BSs are owned by different wireless operators), BSs do not follow fixed rules in ored to avoid subcarrier collisions. Instead, the nature of subcarrier collision (that it can cause mutual damage, but could be avoided by either unilateral or mutual "yielding") can result in "delicate" decision making, which we try to study through a Stackelberg game in this paper. To see this, we first present possible outcomes of the game resulted from different strategy pairs adopted by the BSs, in the following.

\section{Optimization Opportunities and Different Outcomes}

1) Random Allocation: If neither BSs makes an effort to reduce inter-cell interference (i.e., subcarrier collisions), the leftover available subcarriers in $\mathcal{S}_{i}^{A}$ and $\mathcal{S}_{j}^{A}$ will be randomly allocated to MSs in $\mathcal{I}_{i}$ and $\mathcal{I}_{j}$, respectively. Since the subcarrier allocation to MSs outside IZ has already been performed randomly, this outcome is equivalent to the scenario in which subcarriers in the whole cell are randomly allocated.

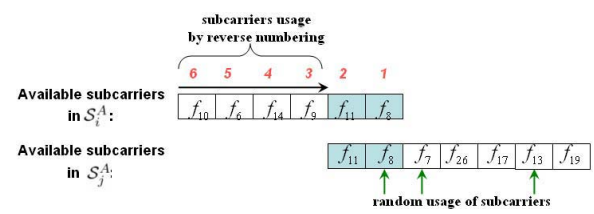

Fig. 2: Blind Optimization performed unilateral by BS $i$.

2) Blind Optimization: When one and only one of the BS (say BS $i$ ) takes effort to reduce possible subcarrier collisions, it first identifies $\mathcal{S}^{O}=\mathcal{S}_{i}^{A} \cap \mathcal{S}_{j}^{A}$ and avoids allocating those subcarriers to MSs in $\mathcal{I}_{i}$, if possible. On the other hand, the other BS (BS $j$ ) will randomly allocate subcarriers in $\mathcal{S}_{j}^{A}$ to MSs in $\mathcal{I}_{j}$. The unilateral effort of interference avoidance by BS $i$ is referred to as "blind optimization". Note that since a subcarrier collision is mutual and equally detrimental to both MSs (from different cells) involved, BS $j$ is in fact "freeriding" the blind optimization performed by BS $i$ in this case.

An example of blind optimization is illustrated in Fig. 2. After initial random allocation, the sets of available subcarriers in two cells are $\mathcal{S}_{i}^{A}=\left\{f_{10}, f_{6}, f_{14}, f_{9}, f_{11}, f_{8}\right\}$ and $\mathcal{S}_{j}^{A}=\left\{f_{19}, f_{13}, f_{17}, f_{26}, f_{7}, f_{11}, f_{8}\right\}$, respectively. The MSs in $\mathcal{I}_{i}$ need 4 subcarriers for their downlink transmission while the MSs in $\mathcal{I}_{j}$ need 3 . Therefore, 3 subcarriers will be randomly chosen from $\mathcal{S}_{j}^{A}$ and allocated to MSs in $\mathcal{I}_{j}$. In 
the example of Fig. 2, these 3 randomly chosen subcarriers are $\left\{f_{7}, f_{8}, f_{13}\right\}$. In contrast, to avoid possible subcarrier collisions from $\mathcal{S}_{j}^{A}$, BS $i$ undergoes blind optimization and identifies that $\mathcal{S}^{O}=\left\{f_{8}, f_{11}\right\}$ and marks those subcarriers with lowest logical numbers $(1,2)$. The subcarrier allocation to MSs in $\mathcal{I}_{i}$ is performed by prioritizing those subcarriers associated with higher logical numbers (or equivalently, to avoid using subcarriers in $\mathcal{S}^{O}$, if possible). In doing so, BS $i$ makes full use of the exchange information (presuming that subcarrier allocation in $\mathcal{I}_{j}$ is random). In Fig. 2, although the $f_{8}$ is randomly chosen by $\mathrm{BS} j$, its usage in $\mathcal{I}_{i}$ is deliberately avoided by BS $i$ and subcarrier collision is prevented.

3) Joint Optimization: In joint optimization, both BSs try to avoid usage of the subcarriers in $\mathcal{S}^{O}$. For example, in Fig. 3 , transmission in $\mathcal{I}_{i}$ requires 5 subcarriers (out of total 6 available subcarriers in $\mathcal{S}_{i}^{A}$ ) while $\mathcal{I}_{j}$ requires 6 (out of 7 available in $\mathcal{S}_{j}^{A}$ ). To avoid subcarrier collision, both BSs mark subcarriers in $\mathcal{S}^{O}$ (i.e., subcarriers $\left\{f_{8}, f_{11}\right\}$ ) with the lowest logical numbers (note that $\left\{f_{8}, f_{11}\right\}$ are marked differently by BS $i$ and BS $j$ ) and both prioritize the usage of subcarriers with higher logical numbers. In this case, the subcarriers $\left\{f_{10}, f_{6}, f_{14}, f_{9}, f_{11}\right\}$ are allocated to $\mathcal{I}_{i}$ while subcarriers $\left\{f_{19}, f_{13}, f_{17}, f_{26}, f_{7}, f_{8}\right\}$ are allocated to $\mathcal{I}_{j}$. As a result, subcarrier collision is prevented by joint effort of both BSs.

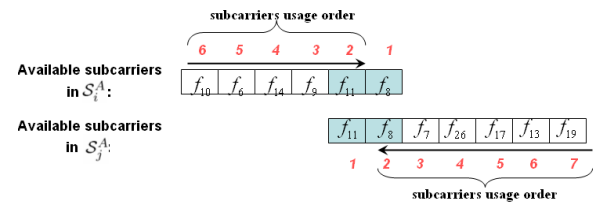

Fig. 3: Joint Optimization performed by both BSs.

III. BINARY STACKELBERG GAME BETWEEN BSS

\section{A. Strategic Game between the Two BSs}

We model the interactions between the two BSs involved in interference management here as a strategic game. In the framework of game theory [9][10], interactive decisionmaking entities (players) evaluate different outcomes of the game using "utilities", which are real numbers that describe the levels of satisfactions a "player" feels toward the outcomes. The outcomes result from strategies taken by all players in the game. A rational player maximizes its own utility by proper decision making. This pursuit of maximum utilities may result in an relatively stable outcome in which all players obtain their "achievable" maximum utilities, given that the actions of other players are fixed. Such an outcome is defined as Nash Equilibrium in game theory literatures and describes the state in which no player can be better off by deviating alone from current outcome. Here we are interested in analyzing how such Nash Equilibrium, if exists, can be achieved in the interference management game played by the two BSs.

TABLE I: Strategic Game Played by Two Base Stations

\begin{tabular}{|c||c|c|}
\hline BS $i$ & Optimize (O) & Not Optimize (NO) \\
\hline \hline Optimize (O) & {$\left[U_{J}^{i}, U_{J}^{j}\right]$} & {$\left[U_{B}^{i}, U_{F}^{j}\right]$} \\
\hline Not Optimize (NO) & {$\left[U_{F}^{i}, U_{B}^{j}\right]$} & {$\left[U_{R}^{i}, U_{R}^{j}\right]$} \\
\hline
\end{tabular}

In the game shown in Table I, each player (i.e., BS $i$ and BS $j$ ) can choose its specific action from the binary strategy space $\mathcal{X}=\{\operatorname{Optimize}(\mathrm{O})$, Not Optimize(NO) $\}$. BS $i$ 's action changes row-wisely while BS $j$ 's action changes column-wisely. The outcome of the game can be denoted as a two-dimensional strategy vector $\mathbf{A}=\left(A_{i}, A_{j}\right)$ such that $\left\{A_{i}, A_{j}\right\} \in \mathcal{X}$. The game results in random allocation when $\mathbf{A}=[\mathrm{NO}, \mathrm{NO}]$; in this case, the utility pair of the two BSs is denoted as $\left[U_{R}^{i}, U_{R}^{j}\right]$, shown by the bottom-right entry of Table I. Similarly, when both BSs agree to participate in mutual interference avoidance (i.e., $\mathbf{A}=[\mathrm{O}, \mathrm{O}]$ ), joint optimization results, which is associated with the utility pair $\left[U_{J}^{i}, U_{J}^{j}\right]$. When (unilateral) blind optimization is performed by one of the BSs, the BS that performs blind optimization achieves utility $U_{B}$ while the other (free-riding) BS achieves utility $U_{F}$, as denoted by the two diagonal entries in Table I.

B. Binary Stackelberg Leader-Follower Game between BSs

The different outcomes in the game shown in Table I result from interactions between BSs and cannot be solely determined by either of the BSs. Therefore, a player has to consider possible response from the other player before making any rational decision. To analyze this decision making process between BSs, we model it as a Stackelberg leaderfollower game in which one of the players (leader) makes decision first before the the other player (follower). The game is binary since each player chooses its action from the binary decision space $\mathcal{X}=\{$ Optimize $(\mathrm{O})$, Not Optimize(NO) $\}$.

\section{Algorithm III.1: BInARY STACKelberg Decision MAKIng ()}

comment: Sequential leader-follower decision making.

initialization: BS $i$ and $j$ exchange information on $\mathcal{S}_{i}^{A}$ and $\mathcal{S}_{j}^{A}$ for BS $i$ :

$$
\text { do }\left\{\begin{array}{l}
\text { predicts } A_{j}^{A_{i}}, A_{i}=\mathrm{O} \text { or } \mathrm{NO} \quad \text { (i) } \\
\text { comment: predicts } \mathrm{BS} j \text { 's optimal response; } \\
A_{i}^{*} \leftarrow \arg \max _{A_{i} \in \mathcal{X}}\left\{U^{i}\left[A_{i}, A_{j}^{A_{i}}\right]\right\} \quad \text { (ii) } \\
\text { comment: } \mathrm{BS} i \text { determines its strategy from prediction } \\
\text { for } \mathrm{BS} j:
\end{array}\right.
$$

comment: BS $j$ makes decision upon observation of $A_{i}^{*}$.

output (utility pair $\left\{U^{i}\left[A_{i}^{*}, A_{j}^{*}\right], U^{j}\left[A_{i}^{*}, A_{j}^{*}\right]\right\}$ )

The "rules" of playing this Stackelberg game is illustrated by Algorithm III.1, in which we assume that BS $i$ is the leader that makes decision first and BS $j$ is the follower ${ }^{3}$. In order to maximize its own utility, BS $i$ first analyzed BS $j$ 's optimal responses to different actions of BS $i$. In the binary Stackelberg game studied here, this analysis reduces the 4 outcomes in Table I to 2 possible outcomes, each of which uniquely associated with BS $i$ 's binary strategy. BS $i$ then chooses the strategy $A_{i}^{*}$ which maximizes its own utility (statement (ii) in Algorithm III.1). BS $j$, on the other hand, observes BS $i$ 's decision and makes its decision based on complete information, to maximize its own utility (statement (iii) in Algorithm III.1) by choosing action $A_{j}^{*}{ }^{4}$ The outcome

\footnotetext{
${ }^{3}$ While the terms "leader" and "follower" may indicate certain superiority of the former over the latter in some cases (e.g., in the Stackelberg duopoly game [9]), they do not carry such implication here. That is, being "leader" does not necessarily imply more leverage over the "follower". Therefore, which BS is the "leader" or "follower" is not of vital importance in this specific game.

${ }^{4}$ When both BSs are rational, $A_{j}^{*}=A_{j}^{A_{i}} \mid A_{i}=A_{i}^{*}$, which was predicated by BS $i$ in statement (ii) in Algorithm III.1.
} 
$\mathbf{A}^{*}=\left[A_{i}^{*}, A_{j}^{*}\right]$ from Algorithm III.1 achieves the unique Nash Equilibrium by definition, since we can see from the decision making process that neither of the two players can deviate alone from this outcome and expect higher payoff. Since the game is played upon (dynamic) initial subcarrier allocation to users outside IZ, the execution of the algorithm would not affect the spectrum usage of those users, in addition, it would not required pre-fixed spectrum allocation either.

\section{Modeling Utility Functions}

In the Stackelberg game described above, the outcome of the decision making process, i.e., statement (i) (iii) in Algorithm III.1, strongly depends on how the utility function is modeled by players. To understand the proper modeling of utility functions, we first investigate here the expected number of subcarrier collisions in different strategic outcomes. To do so, assume that the traffic loads are $\beta_{i}$ and $\beta_{j}$ in the two cell, such that $\left\{\beta_{i}, \beta_{j}\right\} \in[0,1]$, (i.e., neither cell is overloaded). We then have $\left|\mathcal{S}_{i}^{A}\right|=\left[\left(1-\beta_{i}\right)+\beta_{i} f(\alpha)\right] S$, and the number of subcarriers needed by $\mathcal{I}_{i}$ is $\left|\mathcal{S}_{i}^{\mathcal{I}}\right|=S \beta_{i} f(\alpha)$.

1) Expected Number of Subcarrier Collisions in Random Allocation: When neither of the BSs performs any interference avoidance, the expected number of subcarrier collisions in such random allocation can be directly computed as

$$
\mathbb{E}\left[C_{R}\right]=f^{2}(\alpha) \beta_{i} \beta_{j} S .
$$

2) Expected Number of Subcarrier Collisions in Blind Optimization: Without loss of generality, assume that BS $i$ carriers out the unilateral blind optimization while BS $j$ randomly allocates subcarriers. In this case, subcarrier collision is possible when $S_{O}=\left|\mathcal{S}^{O}\right|=\left|\mathcal{S}_{i}^{A} \bigcap \mathcal{S}_{j}^{A}\right|$ is larger than the number of "redundant" subcarriers in cell $i$. We denote the number of subcarriers that are vulnerable to subcarrier collision as $\zeta$; then $\zeta=\left\{\begin{array}{ll}S_{O}-d, & \text { if } n_{B} \geq S_{O}>d ; \\ 0, & \text { otherwise. }\end{array}\right.$, where $d=$ $S\left(1-\beta_{i}\right) \geq 0$ is the number of "redundant" subcarriers in cell $i$. Since $S_{O}$ is governed by the binomial distribution $\mathrm{B}\left(n_{B}, p_{B}\right)$, in which $n_{B}=\left|\mathcal{S}_{i}^{A}\right|=S\left[f(\alpha) \beta_{i}+\left(1-\beta_{i}\right)\right]$ and $p_{B}=f(\alpha) \beta_{j}+\left(1-\beta_{j}\right)$, we can show that $\zeta$ follows a truncated binomial distribution the expected value of which can be computed as

$$
\begin{aligned}
\mathbb{E}[\zeta] & =0+\operatorname{Pr}\left(n_{B} \geq S_{O} \geq d+1\right) \cdot \mathbb{E}\left[S_{O}-d \mid S_{O} \geq d+1\right] \\
& =\mathrm{A}(\cdot)\left[\sum_{k=d+1}^{n_{B}}(k-d)\left(\begin{array}{c}
n_{B} \\
k
\end{array}\right) p_{B}^{k}\left(1-p_{B}\right)^{\left(n_{B}-k\right)}\right] \\
& =\left[\mathbb{E}\left[S_{O}\right] \cdot \mathrm{B}(\cdot)-d \mathrm{C}(\cdot)\right] \mathrm{A}(\cdot),
\end{aligned}
$$

in which $\mathrm{A}(\cdot)=I_{p}\left(d+1, n_{B}-d\right), \mathrm{B}(\cdot)=I_{p}\left(d, n_{B}-d\right)$, and $\mathrm{C}(\cdot)=I_{p}\left(d+1, n_{B}-d\right) ; I_{p}(a, b)=\frac{\int_{0}^{\infty} t^{a-1} /(1+t)^{a+b} d t}{\int_{0}^{1} t^{a-1}(1-t)^{b-1} d t}, \forall\{a, b\} \geq$ 0 is the regularized beta function; $\mathbb{E}\left[S_{O}\right]=n_{B} p_{B}$ is the expected size of $\mathcal{S}^{O}$. Each of the "vulnerable" subcarriers is subject to collision with probability $\frac{\beta_{j} f(\alpha) S}{\left|\mathcal{S}_{j}^{A}\right|}$, from which we can then compute the expected number of subcarrier collisions in blind optimization as

$$
\mathbb{E}\left[C_{B}\right]=\mathbb{E}[\zeta] \frac{\beta_{j} f(\alpha)}{\left(1-\beta_{j}\right)+\beta_{j} f(\alpha)} .
$$

The effectiveness of blind optimization depends (among other things) on the traffic loads in both cells. Particularly, it can be shown that $\frac{\partial \mathbb{E}\left[C_{B}\right]}{\partial \beta_{i}}>0, \forall \beta_{i} \in[0,1]$, which suggests that blind optimization performed by BS $i$ is more effective when the traffic load in cell $i$ is lighter. On the other hand, we can also show that $\frac{\partial \mathbb{E}\left[C_{B}\right]}{\partial \beta_{j}} \lesseqgtr 0$, which indicates that the effectiveness of blind optimization in reducing the subcarrier collision is not monotonic with the traffic load in the other cell. The first observation is quite intuitive since lighter traffic load in cell $i$ leaves BS $i$ with a larger set of $\mathcal{S}_{i}^{A}$ and thus more flexibility to avoid allocating subcarriers in $\mathcal{S}^{O}$ to MSs in $\mathcal{I}_{i}$. The second observation, on the other hand, requires subtler interpretation. As $\beta_{j}$ decreases, the number of subcarriers required by $\mathcal{I}_{j}$ (and thus can cause potential subcarrier collision to MSs in $\mathcal{I}_{i}$ ) decreases. However, decreasing $\beta_{j}$ also enlarges the set $\mathcal{S}_{j}^{A}$, which impairs the ability of BS $i$ to avoid subcarriers allocated to $\mathcal{I}_{j}$ that are randomly chosen from $\mathcal{S}_{j}^{A}$. The joint impact of varying $\beta_{j}$ results from these two conflicting aspects and should be evaluated together from (3) for a rational decision to be made. However, in the special case when $\beta_{i}=\beta_{j}=\beta$, we can simplify (3) and show that $\frac{\partial \mathbb{E}\left[C_{B} \mid \beta_{i}=\beta_{j}=\beta\right]}{\partial \beta}>0$, which is also verified later in Fig. 4 .

3) Expected Number of Subcarrier Collisions in Joint Optimization: Subcarrier collisions only occur (and will inevitably occur) when $\left|\mathcal{S}_{i}^{A}\right|+\left|\mathcal{S}_{j}^{A}\right|-\left|\mathcal{S}^{O}\right|<S f(\alpha)\left(\beta_{i}+\beta_{j}\right)$ in joint optimization, i.e., when the number of distinct subcarriers after the random allocation to $\mathcal{I}_{i}^{C}$ and $\mathcal{I}_{j}^{C}$ is less than the number of subcarriers required by MSs in IZ. We can then write the number of subcarrier collisions as $C_{J}=\left\{\begin{array}{ll}S_{O}-d_{i}+d_{j}, & n_{J} \geq S_{O}>d_{i}+d_{j} \\ 0, & \text { otherwise, }\end{array}\right.$, where $n_{J}=$ $\min \left\{\left|\mathcal{S}_{i}^{A}\right|,\left|\mathcal{S}_{j}^{A}\right|\right\}, d_{i}=\left(1-\beta_{i}\right) S, d_{j}=\left(1-\beta_{j}\right) S$. Let $\mathrm{D}=d_{i}+d_{j}$ and $\gamma_{J}=\max \left\{n_{J}-D, 0\right\}$. Following a discussion similar to the one presented above, the expected number of subcarrier collisions for joint optimization can be given as

$\mathbb{E}\left[C_{J}\right]=0+\operatorname{Pr}\left(n_{B} \geq S_{O} \geq D+1\right) \cdot \mathbb{E}\left[S_{O}-D \mid n_{B} \geq S_{O} \geq D+1\right]$ $=I_{p}\left(D+1, \gamma_{J}\right) \cdot\left(\mathbb{E}\left[S_{O}\right] I_{p}\left(D, \gamma_{J}\right)-D I_{p}\left(D+1, \gamma_{J}\right)\right)$, (4) where $I_{p}(a, b)$ is the regularized beta function. Straightforward computation shows that $\left\{\frac{\partial \mathbb{E}\left[C_{J}\right]}{\partial \beta_{i}}, \frac{\partial \mathbb{E}\left[C_{J}\right]}{\partial \beta_{j}}\right\} \leq 0, \forall\left\{\beta_{i}, \beta_{j}\right\} \in$ $[0,1]$. This meets the intuitive expectations since when both BSs coordinate, the decreasing of traffic load in either cell will enable more effective interference avoidance.

When $\beta_{i}=\beta_{j}=\beta, S=512$ and $R=1$, Fig. 4 evaluates, as a function of $\{\alpha, \beta\} \in[0,1]$, the expected density of subcarrier collision, which is defined as the number of subcarrier collisions normalized by the area of IZ. We can see that with random allocation, considerable amount of subcarrier collisions occur even when the traffic load is relatively low. On the other hand, subcarrier collisions are considerably reduced in blind optimization, in fact, they can virtually be eliminated when $\beta<0.6$ even for $\alpha=1$. Furthermore, when joint optimization is used, further suppression in subcarrier collisions can be observed, e.g., virtually no subcarrier collision occur for a overlapping radius of $\alpha=1$ when $\beta<0.8$. Nevertheless, we should also note that in the extreme case when both cells are fully loaded, there is no room for optimization after the initial random allocation to MSs outside IZ; therefore, all three schemes have identical performance in full-loaded systems.

\section{Modeling Utility by Value and Cost Functions}

In the Stackelberg game described above, the utility function should reflect a rational BS's desire to reduce subcarrier 


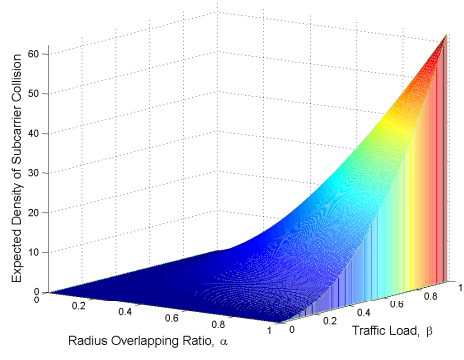

(a) Random Allocation

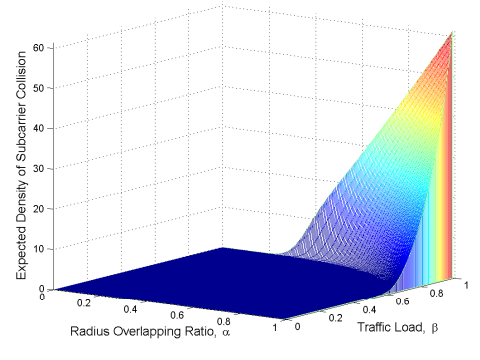

(b) Blind Optimization

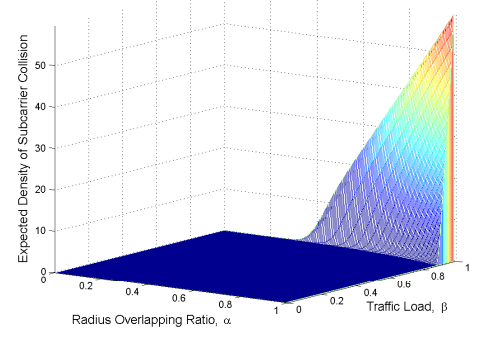

(c) Joint Optimization

Fig. 4: Expected density of subcarrier collisions under different pairs of strategies made by the two BSs. Assume the traffic load in both cells are the same (i.e., $\beta_{i}=\beta_{j}=\beta$ ), and the radius overlapping ratio $\alpha$ ranges from 0 to 1 .

collisions with the minimum cost. More specifically, let $\theta$ be the expected number of subcarrier collisions, the value of an outcome to a BS can be modeled by function $\psi(\theta)$ that monotonically decreases with $\theta$, i.e., $\frac{\partial \psi(\cdot)}{\partial \theta}<0$. Since subcarrier collision is mutual, the value of any outcome $\mathbf{A}$ to both $\mathrm{BSs}$ in the game is equal and is of quantity $\psi\left(\theta_{\mathbf{A}}\right)$, where $\theta_{\mathbf{A}}$ equals to $\mathbb{E}\left[C_{R}\right], \mathbb{E}\left[C_{B}\right]$, or $\mathbb{E}\left[C_{J}\right]$ (which are computed in (2) (4)), depending on the specific outcome $\mathbf{A}$.

In contrast, the cost to achieve a certain outcome $\mathbf{A}$ may differ for different BSs. This is particulary true in the case of blind optimization, where one of the BSs makes a unilateral effort (thereby is incurred with optimization cost) in interference avoidance while the other BSs just free-rides (which costs nothing). To this end, we can model the cost function of BS $i$ in outcome $\mathbf{A}$ to be

$\xi^{i}(\mathbf{A})= \begin{cases}g_{B}(\cdot), & \text { if BS } i \text { performs blind optimization; } \\ g_{J}(\cdot), & \text { if both BS performs joint optimization; } \\ 0, & \text { if BS } i \text { does not engage in optimization }\end{cases}$

The utility of a BS $i$ in the outcome can then be given as

$$
\mathrm{U}^{i}(\mathbf{A})=\psi\left(\theta_{\mathbf{A}}\right)-\xi^{i}(\mathbf{A})
$$

The strategy a BS takes largely depends on the functional form of (5). Specifically, the form of value function $\psi(\cdot)$ and cost function $\xi^{i}(\mathbf{A}) . \psi(\cdot)$ (under the premise that $\frac{\partial \psi(\cdot)}{\partial \theta}<$ $0)$ describes how detrimental is a subcarrier collision. For example, a BS obviously considers the subcarrier collision to be more undesirable when it chooses $\psi(\theta)=\mathrm{e}^{-\theta}$ than when it chooses $\psi(\theta)=\frac{1}{\theta}$, since $\mathrm{e}^{-\theta}<\frac{1}{\theta}, \forall \theta \in \mathfrak{R}^{+}$. The optimization cost can impact the final decision of a BS as well, since BSs are less likely to engage in any optimization if the associated cost is too high.

In Table II we show the fractions of different outcomes in Nash Equilibrium over 10000 observations of the Stackelberg game described by Algorithm III.1. The MSs are randomly distributed and subcarrier is randomly allocated to MSs outside IZ in each observation. Specifically, we assume that BS 1 is the leader and BS 2 is the follower, and that $\beta_{1}=0.98$ while $\beta_{2}$ is governed by a shifted and scaled symmetric Beta distribution with parameter $a=b=3$, mean 0.98 and spans through $[0.96,1]$. The value function is assumed to be $\psi\left(\theta_{\mathbf{A}}\right)=-\theta_{\mathbf{A}}$, while the cost function is simply chosen to be a multi-value function such that $g_{B}(\cdot)=\gamma$ and $g_{J}(\cdot)=2 \gamma$. Here, $\gamma$ is the normalized optimization cost, i.e., the cost of optimization is equivalent to the incurrence of $\gamma$ subcarrier collisions. In Table II, we choose some sample values of $\gamma$; actual values may be chosen based on optimization cost in
TABLE II: Fraction of different outcomes in Nash Equilibrium

\begin{tabular}{|l||c|c|c|c|}
\hline & Random & BO by BS 1 & BO by BS 2 & Joint Opt \\
\hline \hline$\gamma=0$ & $0.0 \%$ & $2.3 \%$ & $3.8 \%$ & $93.9 \%$ \\
$\gamma=1$ & $3.1 \%$ & $11.0 \%$ & $32.4 \%$ & $53.5 \%$ \\
$\gamma=10$ & $97.4 \%$ & $0.9 \%$ & $1.7 \%$ & $0.0 \%$ \\
\hline
\end{tabular}

specific implementations. We can see that when there is no cost in optimization, the strategy $[\mathrm{O}, \mathrm{O}]$, i.e., "Joint Optimization", dominates most of the outcomes, with few exceptions where blind optimization is sufficient for interference avoidance. In the non-ideal case when $\gamma=1$, the outcome of the game begin to vary more. Furthermore, when the optimization cost is unacceptably high $(\gamma=10)$, the best choice for each BS is to avoid the costly optimization and the dominating Nash Equilibrium is the outcome in which both BSs just randomly allocate subcarriers.

This paper describes an adaptive protocol for inter-cell interference management in WiMAX downlink using gametheoretic framework. The protocol requires no system-wise spectrum planning or coordination in spectrum usage from users that are not subject to interference. Intelligent decision makings by BSs in a two-cell model are implemented by an algorithm built upon Stackelberg game. Different pairs of strategies reveal different optimization opportunities in managing interference, through which the Nash equilibria that result from different behaviors of BSs in different system settings are examined.

\section{REFERENCES}

[1] "IEEE Standard for Local and Metropolitan Area Networks - Part 16: Air Interface for Fixed Broadband Wireless Access Systems," October 2004;

[2] "IEEE Standard for Local and Metropolitan Area Networks - Part 16: Air Interface for Fixed Broadband Wireless Access Systems, Amendment 2,'February 2006;

[3] Huawei, "3GPP R1- 050507: Soft Frequency Reuse Scheme for UTRAN LTE," TSG RAN WG1 Meeting 41, Athens, Greece, May. 2005;

[4] Siemens, "3GPP: R1-060135: Interference Mitigation by Partial Frequency Reuse," TSG-RAN WG1 Ad Hoc Meeting on LTE, Helsinki, Finland, January 2006;

[5] M. Sternad, T. Ottosson, A. Ahlen, A. Svensson, "Attaining both Coverage and High Spectral Efficiency with Adaptive OFDM Downlinks," IEEE Vehicular Technology Conference, Orlando, Oct. 2003.

[6] Y. Xiang, J.Luo and C. Hartmann, "Inter-cell Interference Mitigation through Flexible Resource Reuse in OFDMA based Communication Networks", Proceedings of European Wireless Conference, 2007.

[7] J.H Yeom, Y.H. Lee, "Mitigation of inter-cell interference in the WiMAX system," IEEE Mobile WiMAX Symposium, March 2007 Page(s):26 - 31;

[8] I. Katzela and M. Naghshineh, "Channel Assignment Schemes for Cellular Mobile Telecommunication Systems: a Comprehensive Survey,' IEEE Personal Communications, vol. 3, no. 3, pp. 10-31, June 1996;

[9] Martin J. Osborne, An introduction to game theory, Oxford University Press, August 2003.

[10] Y. Chen and S. Kishore, "A Game Theoretic Study of Cooperative Communication," IEEE Trans. on Wireless Communications (to appear); 